\title{
Evaluación de los factores de riesgo y los tipos de superficie para el desarrollo de las úlceras por presión en el enfermo crítico
}

\author{
Assessment of risk factors and the types of surface for the development of pressure \\ ulcers on critical ill patients
}

${ }^{1}$ Concepció Fuentes i Pumarola

${ }^{2}$ Núria Bisbe Company

${ }^{2}$ Maria Àngels Galvany Ferrer

${ }^{2}$ Dolors Garangou Llenas

Correspondencia:

Concepció Fuentes i Pumarola

Departamento de Enfermería UdG

Emili Grahit, 77

17071 Girona

Tfno.: 972418770

E-mail: concepcio.fuentes@udg.es
'DUE. Profesora colaboradora Escuela Universitaria de Enfermería, Universidad de Girona. ${ }^{2}$ DUE. Servicio de Medicina Intensiva, Hospital Universitario de Girona Dr. Josep Trueta.

\section{RESUMEN}

Objetivos: Evaluar los factores de riesgo y los tipos de superficie para el desarrollo de las úlceras por presión (UPP) en el enfermo crítico ingresado en una unidad de cuidados intensivos (UCI). Material y métodos: Estudio prospectivo de incidencia de UPP. Población: Enfermos ingresados en una UCI con EMINA ${ }^{\circ}$ de alto riesgo, estancia mínima de 48 horas y que no presentaran UPP al ingreso. Se registraron los datos demográficos, de gravedad al ingreso (SAPS II y APACHE III), diabetes, estado séptico, ventilación mecánica, incidencia y grado de UPP, y tipo de superficie de apoyo (alternante [SA] o estática [SE]). Conclusiones: Los factores de riesgo asociados con el desarrollo de UPP en el enfermo crítico fueron la edad, la estancia media y el tipo de superficie de apoyo, siendo muy significativa la disminución de la incidencia de UPP con las superficies alternantes.

\section{PALABRAS CLAVE}

Úlceras por presión, factores de riesgo, incidencia, escala de EMINA, cuidados críticos, superficies de apoyo.

\section{INTRODUCCIÓN}

De acuerdo con las directrices generales sobre prevención de úlceras por presión (UPP) del Grupo Nacional para el Estudio y Asesoramiento en Úlceras por Presión y Heridas Crónicas (GNEAUPP) (1), las UPP constituyen un verdadero problema en diferentes ámbitos, como la salud y la calidad de vida de los pacientes, comportando un aumento de los recursos para el sistema de salud tanto humanos como materiales. La mayoría de ellas se podrían prevenir con unas estrategias de educación y prevenciones protocolizadas (2). Este problema es 


\section{SUMMARY}

Objectives: To assess the risk factors and the types of surface for the development of pressure ulcers (PU) on critical ill patients in an Intensive Care Unit (ICU). Material and methods: Prospective study about the incidence of PU. Population: All patients on ICU with EMINA ${ }^{\circledR}$ of high risk, who stay 48 h. at least and don't have PU in the moment of admission. Information about demographic characteristics, gravity in the moment of admitting was recorded: SAPS II and APACHE III, diabetes, sepsis, mechanical ventilation, incidence and graduation of $P U$, and the type of support surface: alternate (AS) or static (SS). Conclusions: The risk factors associated with the development of $P U$ on critical ill patients were the age, the average time in ICU and the type of support surface, being the reduction on incidence of $P U$ with the alternate surfaces very important.

\section{KEY WORDS}

Pressure sores, risk factors, incidence, EMINA score, critical care, support surfaces.

común en muchas instituciones sanitarias de toda Europa (3) y puede comportar responsabilidades legales al ser en muchas situaciones un problema evitable. En el Reino Unido las repercusiones legales del problema de las UPP ya son evidentes (4).

La prevalencia de UPP en las unidades de críticos en el Estado español es del 13,16\% (5). Las localizaciones más frecuentes son: sacro $36,9 \%$, talón 28,35\%, occipital 13,04\% (6).

La prevalencia en las unidades de críticos del Hospital Universitario de Girona Dr. Josep Trueta en el año 2003 fue del 19,37\%.

En los hospitales del Instituto Catalán de la Salud (ICS) se hace una monitorización de incidencia y prevalencia de las UPP como control de calidad de los cuidados de enfermería. La monitorización de la incidencia de las UPP es importante para la prevención de su aparición (7), así como utilizar una buena escala de valoración del riesgo potencial. Los requisitos básicos de una escala de valoración son que los ítems sean sensibles, específicos, aplicables y variables. En nuestro hospital utilizamos la escala EMINA (8) (Anexo 1), que ha sido elaborada por un grupo de enfermeras expertas en cuidados, gestión e investigación. Es una escala validada en todos los hospitales del ICS a través de un proyecto de investigación coordinado y financiado por el Fondo de Investigación Sanitaria (FIS).

En nuestra unidad observamos que en pacientes con el mismo riesgo (al- to) de desarrollar UPP según la escala EMINA, a los que se aplicaban los mismos cuidados de prevención, algunos las desarrollaban, siendo la única diferencia la superficie de apoyo debido a que sólo disponíamos de siete superficies dinámicas (colchones alternantes de aire). El resto de camas estaban dotadas de superficies estáticas (colchonetas de fibras siliconizadas, colchones de espuma viscolástica o colchones de foam de poliuretano).

Diversos estudios centran la disminución de incidencias de UPP con la utilización de superficies adecuadas (9, 10).

La principal diferencia entre las superficies estáticas y las dinámicas es que las primeras nunca alcanzan presiones por debajo de los 17-20 mm $\mathrm{Hg}$, mientras que las dinámicas pueden alcanzar valores inferiores a éstas gracias a la alternancia de llenado y vaciado de celdas de aire. Esto permite reducir y aliviar la presión en zonas de contacto. Los niveles de presión dependen del tamaño de sus celdas y su efectividad se mide según el tiempo de duración de cada ciclo (alrededor de 10 minutos). Este tipo de superficies está indicado para pacientes de medio y alto riesgo de UPP. Muchos autores han encontrado una relación significativa entre la severidad de las escalas de gravedad y el desarrollo de las úlceras por presión $(11,12,13)$. Por todo ello, se planteó esta investigación con los siguientes objetivos: evaluar posibles factores de riesgo que no se contemplan en las escalas de gra- vedad para el desarrollo de las úlceras por presión y valorar la influencia de los tipos de superficie utilizadas en el enfermo crítico ingresado en nuestra unidad de cuidados intensivos.

\section{MATERIAL Y MÉTODOS}

Se trata de un estudio prospectivo de incidencia de UPP en enfermos críticos con EMINA de alto riesgo y gravedad severa con un NEMS (Nine Equivalents of Nursing Manpower use Score) medio de 32 puntos, donde se analizan los factores de riesgo, los parámetros de gravedad y la puntuación EMINA en aquellos enfermos que desarrollan UPP.

El estudio lo realizó el personal de enfermería en la UCI polivalente (14 camas) del Hospital Universitario de Girona Dr. Josep Trueta, hospital de nivel 2. Tomamos como muestra todos los enfermos ingresados en la UCI entre enero y septiembre de 2005. Los criterios de inclusión fueron: enfermos ingresados con EMINA de alto riesgo: 8-15 puntos y una estancia mínima de 48 horas, siendo los criterios de exclusión los enfermos que presentaban una UPP al ingreso en la unidad.

Las variables estudiadas fueron: edad, sexo, estancia media, grupo diagnóstico, si padecían diabetes, si se encontraban en estado séptico (14) (Anexo 2), si precisaban ventilación mecánica, índices de gravedad (SAPS II, APACHE III), puntuación escala EMINA, incidencia de úlceras, fecha de aparición UPP, localización y grado, tipo de superficie de apoyo (estática o dinámica). Durante el período de nuestro estudio disponíamos de los siguientes colchones alternantes como superficies dinámicas: un colchón Trinova $^{\circledR}$, un Nimbus ${ }^{\circledR}$ 3, dos Alpha Relief $^{\circledR}$, un Aerocare ${ }^{\circledR}$ 2010, un Duo ${ }^{\text {тм}} 2$ y un TheraKair ${ }^{\circledR}$. El resto de camas estaban dotadas de las superficies estáticas: una colchoneta de fibras siliconizadas, tres colchones de espuma viscolástica, y tres colchones de foam de poliuretano. Los enfermos que ingresaban ocupaban la cama libre que había en ese momento y por tanto con la superficie de apoyo disponible en la cama (dinámica o estática). 
Para todos los pacientes se siguió el protocolo establecido en nuestro hospital, revisado en noviembre de 2004, que tiene en cuenta los factores de riesgo descritos para el desarrollo de UPP y las recomendaciones de la GNEAUPP, EPUAP y Grupo de Enfermería del ICS para evitar los factores extrínsecos $(1,3,8,15)$.

Se elaboró una hoja para la recogida de datos (Anexo 3). Iniciábamos la recogida de datos a las 48 horas de ingresar el enfermo que reunía los criterios de inclusión antes descritos. Valorábamos el estado de la piel del enfermo diariamente. En caso de aparición de UPP se registraba el día, grado y localización. Finalizábamos la recogida de datos en caso de aparición de UPP, al alta o si se producía defunción del paciente.

El análisis estadístico de los datos recogidos se realizó con el paquete estadístico SPSS versión 12. Las variables cuantitativas se expresaron con la media (DE) y las cualitativas mediante porcentajes. Se compararon ambos grupos con la t de Student y $\chi^{2}$. Se consideró que existía significación estadística cuando se halló un valor de $\mathrm{p}<0,05$.

\section{RESULTADOS}

Se incluyeron en el estudio 210 enfermos de los cuales el 64,3\% eran hombres y el 35,7\% mujeres. El 39,5\% de éstos dispusieron de superficies dinámicas mientras que el $60,5 \%$ de su- perficies fueron estáticas (Fig. 1). La edad media de la muestra fue de 54 (DE 19) años (mín. 14 - máx. 86). La distribución por grupos diagnósticos fue la siguiente: el 32,4\% eran enfermos quirúrgicos, el $37,6 \%$ médicos, y el 30\% traumáticos. La media de la puntuación EMINA fue de 11,57 (DE 1) puntos. La estancia media de los pacientes estudiados fue de 12,29 (DE 9) días (mín. 2 - máx. 56). Desarrollaron UPP un total de 43 enfermos (20,5\%), el $16,3 \%$ disponía de superficies dinámicas y el $83,7 \%$ de superficies estáticas ( $p<0,0005)$ (Fig. 2). En la Tabla 1 se resume la localización y estadios de las UPP desarrolladas. La media de días de aparición de UPP fue de 6,26 (DE 5,14) días, la compara-

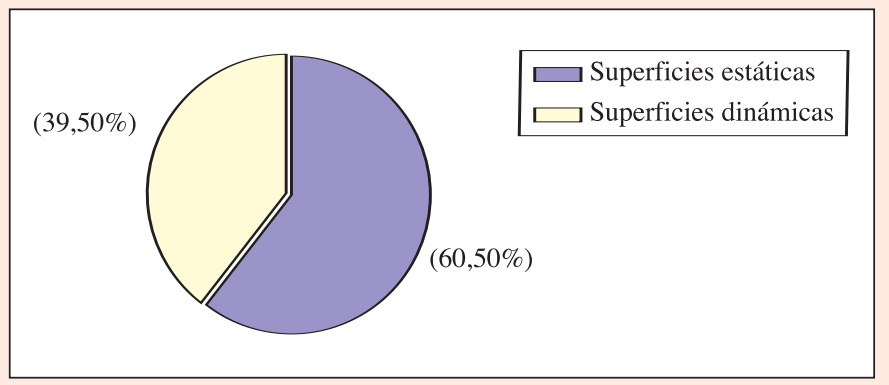

Fig. 1. Distribución de la muestra según tipo de superficies de apoyo.

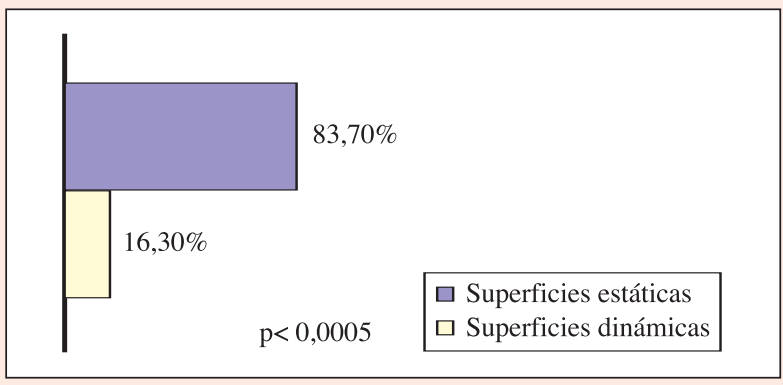

Fig. 2. Distribución de UPP según superficies de apoyo.
Tabla 1. Localización y estadios de las UPP desarrolladas

\begin{tabular}{|l|c|c|c|c|}
\hline Localización & Frecuencia & Porcentaje & $\begin{array}{c}\text { Estadio I } \\
\text { (n) }\end{array}$ & $\begin{array}{c}\text { Estadio II } \\
\text { (n) }\end{array}$ \\
\hline 1. Sacro & 19 & $44,19 \%$ & 14 & 5 \\
2. Talón & 7 & $16,28 \%$ & 6 & 1 \\
3. Glúteo & 7 & $16,28 \%$ & 6 & 1 \\
4. Trocánter & 5 & $11,63 \%$ & 3 & 2 \\
5. Codo & 1 & $2,33 \%$ & - & 1 \\
6. Tibia & 1 & $2,33 \%$ & - & 1 \\
7. Rodilla & 1 & $2,33 \%$ & - & 1 \\
8. Occipital & 1 & $2,33 \%$ & - & 1 \\
9. Maleólo & 1 & $2,33 \%$ & - & 1 \\
TOTAL & 43 & $100 \%$ & 29 & 14 \\
\hline
\end{tabular}

Tabla 2. Relación sexo, grupo diagnóstico y aparición de UPP

\begin{tabular}{|l|l|c|c|c|}
\hline & & $\begin{array}{c}\text { Con UPP } \\
\mathbf{( \% )}\end{array}$ & $\begin{array}{c}\text { Sin UPP } \\
(\mathbf{\%})\end{array}$ & $\begin{array}{c}\mathbf{p} \\
\text { (significación) }\end{array}$ \\
\hline Sexo & Mujer $(\mathrm{n}=75)$ & $37,2 \%$ & $35,3 \%$ & $\mathrm{p}=0,819$ \\
& Hombre $(\mathrm{n}=135)$ & $62,8 \%$ & $64,7 \%$ & \\
Grupo & Quirúrgico (n=68) & $41,9 \%$ & $29,9 \%$ & $\mathrm{p}=0,225$ \\
diagnóstico & Médico ( $=79)$ & $37,2 \%$ & $37,7 \%$ & \\
& Traumático (n=63) & $20,9 \%$ & $32,3 \%$ & \\
& & & & \\
\hline
\end{tabular}

ción de medias según el tipo de superficie no resultó estadísticamente significativa $(p=0,885)$. La edad media de los pacientes con UPP fue de 59,8 (DE 19) años y de 52,6 (DE 18) años en los enfermos que no desarrollaron UPP $(p=0,02)$. La estancia media de los enfermos con UPP fue de 19,4 (DE 12) días y de 10,4 (DE 7) días en los que no desarrollaron úlcera $(\mathrm{p}<0,0001)$. No hallamos diferencias significativas entre la aparición de UPP, sexo y grupo diagnóstico (Tabla 2). Tampoco hallamos diferencias entre UPP, diabetes, estado séptico, ventilación mecánica y los índices de gravedad (SAPS II y APACHE III) (Tablas 3 y 4$)$.

\section{DISCUSIÓN}

Los resultados muestran que de los posibles factores de riesgo analizados que no se contemplan en las escalas de gravedad para el desarrollo de las UPP, como la diabetes, ventilación 
mecánica, estado séptico, índices de gravedad (SAPS II y APACHE III), ninguno presentó una relación estadísticamente significativa en nuestro estudio.

Existen diferentes escalas de valoración de riesgo para desarrollar UPP. Aunque, según Keller y cols. (16) y Westrate y cols. (17), las escalas de Braden y la de Waterlow son las únicas testadas científicamente para ser utilizadas en la UCI, observamos que en la escala de Waterlow el ítem de la nutrición es valorado sin tener en cuenta los valores séricos proteicos al igual que la escala Braden. En nuestro trabajo utilizamos la escala EMINA, donde este ítem sí se tiene en cuenta, por

\begin{tabular}{|l|l|r|r|r|}
\hline \multicolumn{5}{|c|}{ Tabla 3. Relación entre factores de riesgo y aparición de UPP } \\
\hline & & Con UPP & Sin UPP & p (significación) \\
\hline Diabetes & & & & \\
& Sí $(\mathrm{n}=34)$ & $26,5 \%$ & $73,5 \%$ & $\mathrm{p}=0,344$ \\
Estado séptico & No (n=167) & $19,3 \%$ & $80,7 \%$ & \\
& Sí (n=90) & $25,6 \%$ & $74,4 \%$ & $\mathrm{p}=0,114$ \\
Ventilación mecánica & No (n=120) & $16,7 \%$ & $83,3 \%$ & \\
& Sí (n=194) & $21,6 \%$ & $78,4 \%$ & $\mathrm{p}=0,142$ \\
& No (n=16) & $6,3 \%$ & $93,6 \%$ & \\
\hline
\end{tabular}

Tabla 4. Relación entre medias de índices de gravedad y aparición de UPP

\begin{tabular}{|l|c|c|c|}
\hline & Con UPP & Sin UPP & \\
\hline APACHE III & $70,36(\mathrm{DE} 19,363)$ & $63,66(\mathrm{DE} 25,557)$ & $\mathrm{p}=0,114$ \\
SAPS II & $45,15(\mathrm{DE} 11,936)$ & $40,66(\mathrm{DE} 14,812)$ & $\mathrm{p}=0,067$ \\
\hline
\end{tabular}

\begin{tabular}{|clcccc|}
\hline \multicolumn{5}{c}{ ANEXO 1. Escala EMINA (8): valoración de riesgo } \\
Puntos & Estado mental & Movilidad & Incontinencia & Nutrición & Actividad \\
$\mathbf{0}$ & Orientado & Completa & No & Correcta & Deambula \\
$\mathbf{1}$ & Desorientado, & Ligeramente & Urinaria o fecal & Ocasionalmente & Deambula \\
& apático o pasivo & limitada & ocasional & incompleta & con ayuda \\
$\mathbf{2}$ & Letárgico o hipercinético & Limitación & Urinaria o fecal & Incompleta & Siempre \\
& & importante & habitual & & precisa ayuda \\
$\mathbf{3}$ & Comatoso & Inmóvil & Urinaria y fecal & No ingesta o proteínas & No deambula \\
& & & séricas bajas & \\
& & & &
\end{tabular}

\section{ANEXO 2. Estado séptico (14)}

SRIS: Respuesta inflamatoria sistémica a una lesión o agresión, con independencia de su causa, manifestada por una o más de las siguientes características:

1. Temperatura $>38^{\circ} \mathrm{C} \mathrm{o}<36^{\circ} \mathrm{C}$.

2. Frecuencia cardíaca $>90$ l. p. m.

3. Taquipnea manifestada por una frecuencia respiratoria $>$ a 20 r.p.m. o por hiperventilación indicada por un valor de $\mathrm{PaCo} 2<32 \mathrm{~mm} \mathrm{Hg}$

4. Recuento anómalo de leucocitos $>12.000 \mathrm{cel} . / \mathrm{mm}^{3}$, $<4.000 \mathrm{cel} . / \mathrm{mm}^{3}$ o presencia de $>10 \%$ de neutrófilos inmaduros.

SEPSIS: SRIS derivado de una infección (bacteriana, vírica, micótica o parasitaria).

SEPSIS GRAVE: Sepsis asociada como mínimo a una disfunción orgánica aguda, hipoperfusión, hipotensión.

SHOCK: Hipotensión inducida por la sepsis que persiste a pesar de la reposición adecuada de líquidos.

SDMO: Síndrome de disfunción multiorgánica. Alteración de la función de uno o más órganos de un paciente crítico, donde la homeostasis no se puede mantener sin intervención.
ANEXO 3. Hoja de recogida de datos

Número de caso:

Fecha ingreso:

Fecha alta:

Alta $\square \quad$ Éxitus $\square$

Diagnóstico ingreso:

Puntuación EMINA

Diabetes:

Estado séptico: $\square$ Sí $\square$ No

Ventilación mecánica: $\square$ Sí $\square$ No

Puntuación APACHE III:

Puntuación SAPS II:

Superficie alternante: $\square$ Sí $\square$ No

Aparición UPP:

$\square$ Sí $\square$ No

Día aparición UPP:

Localización UPP:

Grado: 
lo que la consideramos válida para los enfermos críticos.

El sexo, en los resultados obtenidos en nuestro estudio, no se correlaciona con la incidencia de UPP como observan Aizpitarte y cols. (18). Al contrario que estos autores, en nuestros resultados la edad sí se relaciona con la incidencia de UPP. Arrondo y cols. (19), en su estudio de 1995 coinciden con nuestros resultados respecto a la asociación estadísticamente significativa entre las variables edad y estancia con la aparición de UPP.

En el análisis de los resultados se observa la eficacia de las superficies de apoyo dinámicas en la prevención de la aparición de UPP, igual que se describe en otros estudios (Torrance y cols. (7), Keller y cols. (16) y Ramón y cols.
(10). Sin embargo, otros autores (Gozalbes y cols. 2005 (20), en su revisión bibliográfica concluyen que no disponen de evidencia de suficiente calidad que permita recomendar las superficies de aire alternante sobre otras superficies. Pero dichos estudios contemplan pacientes con diferentes valoraciones de riesgo y de diferentes ámbitos, así como con tamaños de muestra a veces insuficientes, mientras que nuestro estudio se centra sólo en enfermos críticos con alto riesgo.

\section{CONCLUSIONES}

Los factores de riesgo asociados con el desarrollo de UPP en el enfermo crítico fueron: la edad, la estancia media y el tipo de superficie de apoyo; siendo muy significativa la disminu- ción de la incidencia de UPP con las superficies dinámicas alternantes de aire.

Los resultados de este estudio han conllevado la instauración de colchones alternantes de aire para todas las camas en la nueva UCI de nuestro hospital. Evidentemente la utilización de superficies dinámicas en ningún caso debe sustituir otras medidas de prevención como son las que contempla nuestro protocolo.

\section{AGRADECIMIENTOS}

Quisiéramos agradecer la colaboración de Marga Montiel, DUE, por su participación en la recogida de datos, a los Dres. Alfons Bonet y Josep-Maria Sirvent por su apoyo y asesoramiento a lo largo del estudio.

\section{BIBLIOGRAFÍA}

1. GNEAUPP. Directrices generales sobre prevención de úlceras por presión. Documentos del GNEAUPP. (Actualizado en 2002; citado el 28 noviembre de 2003). Disponible en: www.gneaupp.org

2. Krouskop TA, Noble PC, Garber SL, Spencer WA. The efectiviness of preventive management in reducing the ocurrence of pressure sores. J Rehab (R\&D) 1983; 29: 74-83.

3. European Pressure Ulcer Advisory Panel. Guidelines on treatment of pressure ulcers. EPUAP Review 1999; 1: 31-3.

4. Chapman ND. Repercusiones legales del problema de las úlceras por presión en el Reino Unido. Mesa de Debate: Las Úlceras por Presión, un reto para el sistema de salud y la sociedad: repercusiones a nivel epidemiológico, ético, económico y legal. GENEAUPP. Serie Documentos de Debate, 2003; p. 24-6.

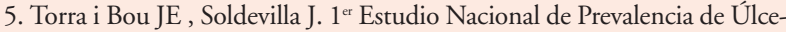
ras por Presión en España. Epidemiología y variables definitorias de las lesiones y pacientes. 2001 Mesa de Debate: Las Úlceras por Presión, un reto para el sistema de salud y la sociedad: repercusiones a nivel epidemiológico, ético, económico y legal. GENEAUPP. Serie Documentos de Debate, 2003; p. 6-14.

6. Torra i Bou JE, Rueda López J, Soldevilla Agreda JJ, Martínez Cuervo F, Verdú Soriano J, GENEAUPP. 1er Estudio Nacional de Prevalencia de Úlceras por Presión en España. Beca Huntleigh Healthcare-UIFC 2001. Gerokomos 2003.

7. Torrance C, Maylor M. Pressure sore survey: Part One. J Wound Care 1999 Jan; 8 (1): 27-30.

8. Grup d'infermeria de l'Institut Català de la Salut per al seguiment de les nafres per pressió. EMINA. Cures d'infermeria per a la prevenció i el tractament de les nafres per pressió. Pagès Editors, 2002.

9. Cullum N, Nelson E, Nixon J. Pressure Sores. Clin Evid 2003 jun; (9): 2167-76.

10. Ramón Cantón C, Salvador Guadayol C, Torra i Bou JE. Úlceras por presión: evaluación de la utilización sistemática de un parque de superficies especiales para el manejo de la presión en la Unidad de Cuidados Intensivos del Hospital de Tarrasa. Enfermería Intensiva 2000; 11 (3):118-26.

11. Wille J. Prevention of pressure sores in surgical patients with emphasis on intensive care patients. Thesis 1998. University of Utrecht, Utrecht.

12. Clough. The cost of pressure area management in an intensive care unit. J Wound Care 1994; 3: 33-45.

13. Inman KJ, Dymock K, Fysh N, Robbins B, Ruledge FS, Sibbald WJ. Pressure ulcer prevention: a randomized controlled trial of risk-directed strategies for patient surface assigment. Adv Wound Care 1999; 12: 72-80.

14. Bone RC, Balk RA, Cerra FB. Definitions for sepsis and organ failure and guidelines for the use of innovative therapies in sepsis. The ACCP/SCCM Consensus Conference Committee. American College of Chest Physicians/Society of Critical Care Medicine. Chest 1992; Jun; 101 (6):1644-55.

15. García Fernández FP, Pancorbo Hidalgo PC, Laguna Parras JM. Guía para el cuidado del paciente con úlceras por presión o con riesgo de desarrollarlas. Hospital universitario "Princesa de España". Servicio Andaluz de Salud. Consejería de Salud. Junta de Andalucía, 2001.

16. Keller BP, Wile J, van Ramshorst B, van der Werken C. Pressure ulcers in intensive care patients: a review of risks and prevention. Intensive Care Med 2002: 28: 1379-88.

17. Westrate JT, Hop WC, Aalbers AG, Vreeling AW, Bruining HA. The Clinical relevance of the Waterlow pressure sore risk scale in the ICU. Intensive Care Med 1998; 24: 815-20.

18. Aizpitarte Pegenaute E, García de Galdiano Fernández A, Zugazagoitia Ciarrusta N, Margall Coscojuela A, Aisiain Erro MC. Úlceras por presión en cuidados intensivos: valoración del riesgo y medidas de prevención. Enfermería Intensiva 2005; 16 (4): 153-63.

19. Arrondo Díez I, Huizi Egileor X, Gala de Andrés M, et al. Úlceras por decúbito en UCI. Análisis y atención de enfermería. Enf Intensiva 1995; 6: 156-64.

20. Gozalbes MA, Villegas R. Agencia de evaluación de tecnologías Sanitarias de Andalucía. Efectividad de las "superficies de alivio de presión" en la prevención y tratamiento de las úlceras por presión. "Colchones de presión de aire alternante". Sevilla, abril 2005. Informe 5/2005. Junta de Andalucía. 